\title{
Shear bond strength of three diferent bonding systems for orthodontic brackets
}

Luís Filipe Siu Lon', Luegya Amorim Henriques Knop', Ricardo Lima Shintcovsk', Odilon Guariza Filho², Dirceu Barnabé Raveli ${ }^{3}$

${ }^{1}$ DDS, MS, Department of Orthodontics, São Paulo State University (UNESP), School of Dentistry, Araraquara, Brazil.

${ }^{2}$ Professor, Graduate Program in Orthodontics, School of Health and Biosciences, PontifICIAL CATHOLIC UniversiTY OF Paraná, Brazil.

${ }^{3}$ Adjunct Professor of Orthodontics, São Paulo State University (UNESP), School of Dentistry, Araraquara, Brazil.
Corresponding author: Dr. Luís Filipe Siu Lon Rua Ébano Pereira, 60, sl 1105 Curitiba - Paraná CEP: 80410-902 - Brazil Phone: 55-41-99838-1718/ Fax Number: 55-41 3322-5776 e-mail: filipelon@hotmail.com

Received: December 05, 2017

Accepted: June 02, 2018
Aim: The objective of this study was to compare the efficiencies of different adhesive systems used to bond orthodontic brackets as well as the fracture pattern during debonding on bovine teeth. Methods: The sample included 45 specimens assigned to 3 groups according to the adhesive system applied: Group I: Transbond XT (3M Unitek ${ }^{\oplus}$, Monrovia/CA - USA), Group II: Orthocem (FGM ${ }^{\circledR}$ Joinville/ SC-BR), and Group III: Orthobond (Morelli®, Sorocaba/SP BR). For this purpose, metal brackets were bonded to bovine teeth following the instructions from each manufacturer. The specimens were subjected to a shear test to assess bond strength (BS). Finally, after debonding, the adhesive remnant index (ARI) was estimated. Results: The average shear strength for a tooth bonded using the adhesive system Transbond XT was $16.39 \mathrm{MPa}$, while it was 18.08 Mpa for Orthocem and 7.28 Mpa for Orthobond; The Tukey test revealed no statistically significant differences between groups I and II $(p<0.01)$ and group III differed statistically from groups I and II. Conclusion: In conclusion, both adhesive systems Transbond XT and Orthocem attained higher bond strength values than Orthobond; the fracture pattern was similar for all adhesive systems applied.

Keywords: Dental bonding. Shear strength. Dental enamel. 


\section{Introduction}

Brackets are essential pieces of orthodontic appliances. Direct bonding is an effective alternative to the procedure of welding bands, which represents a true revolution in orthodontic treatments.

Direct bonding features several advantages, such as better appearance, easy manipulation, enhanced comfort of the patient, absence of pain, decreased irritation of the soft tissue, easier oral hygiene, decreased incidence of gingivitis, better caries detection, possibility of exact bracket positioning, and no space closure required after the treatment. The primary disadvantage is decreased retention area, which directly interferes with masticatory force resistance resulting in accidental debonding and subsequent professional frustration, longer procedures and higher costs ${ }^{1-4}$.

Previous studies have commonly explored factors regarding the optimization of bond strength. Retention mechanisms present in the brackets, the adhesive system, and the acid conditioning of the enamel can influence bond strength. Insufficient adhesive strength is considered a cause of bracket debonding ${ }^{1,25-8}$.

For the past few years, significant scientific and technological advances have brought several benefits to Orthodontics especially in the area of direct bonding of the accessories. New techniques and materials have been incorporated into clinical practice leading to simpler procedures and improved comfort of a patient. Despite the great advances in the development of orthodontic adhesives, studies have shown a failure rate of 5 to $7 \%$ in resin-based bonding 5 .

Orthodontic clinics have been applying fluidic systems immediately after the acid etching of enamel using no intermediate adhesives. As the number of steps during bonding is reduced, orthodontists can decrease chair time and potential errors associated with the contamination during bonding procedures ${ }^{9}$. One example of fluidic systems is Orthocem ( $\mathrm{FGM}^{\circledR}$, Joinville/SC).

The most frequently used adhesive systems for bonding orthodontic brackets in the Brazilian dental market are Transbond XT (3M Unitek ${ }^{\circledR}$, Monrovia/USA), Orthocem (FGM ${ }^{\circledR}$, Joinville/SC-BR), and Orthobond (Morelli®, Sorocaba/SP-BR). Although published scientific studies comparing either Orthocem or Orthobond with other adhesive systems are available in the literature ${ }^{1,10-15}$, to our knowledge, no experiments comparing these two adhesive systems to each other have been conducted to date.

The objective of this study was to compare the shear bond strength (SBS) and the adhesive remnant index (ARI) between 3 different adhesive systems: Transbond XT (3M Unitek ${ }^{\oplus}$, Monrovia/USA), Orthocem ( $F G M{ }^{\circledast}$, Joinville/SC-BR), and Orthobond (Morelli®, Sorocaba/SP-BR). The hypothesis tested in this study was that there are no significant differences in bond strength among the bonding materials.

\section{Material and methods}

This study was approved by the Ethics Committee in the Use of Animals (CEUA) of the Pontificia Universidade Catolica do Paraná (protocol number: 204/07). 
The sample was composed of 45 freshly extracted bovine permanent incisors randomly divided into 3 groups $(n=15)$ :

Group I (control) - 15 brackets by Miniature Twin (3M Unitek ${ }^{\circledR}$, Monrovia/USA) bonded with resin Transbond XT (3M Unitek ${ }^{\circledR}$, Monrovia/USA); Group II - 15 brackets by Miniature Twin (3M Unitek ${ }^{\circledR}$, Monrovia/CA - USA) bonded with resin Orthocem (FGM ${ }^{\circledR}$, Joinville/SC - BR); Group III - 15 brackets by Miniature Twin (3M Unitek ${ }^{\circledR}$, Monrovia/ CA-USA) bonded with resin Orthobond (Morelli®, Sorocaba/SP-BR).

The coronary portion of the teeth selected for this study was characterized by integrity of the crowns, the absence of decay, cracks or fractures to ensure even quality of the sample. To prepare the specimens, each tooth presenting flat surface was selected and attached to a mechanical workbench by clamping (Metalsul ${ }^{\circledR}$, Joinville/SC-BR). Each randomly selected tooth was submitted to prophylaxis with water and pumice stone using a rubber cup (Microdont, São Paulo/SP-BR), and the enamel surface was rinsed with distilled water and dried with oil-free compressed air for 20 seconds. A new rubber cup was used every 5 prophylaxis cycles. A 37\% phosphoric acid gel (Condac, FGM ${ }^{\circledast}$, Joinville/SC-BR) was applied to the buccal surface of each tooth for 30 seconds for enamel etching, followed by air/water spray rinse for 10 seconds and gentle drying with airflow for 10 seconds. The adhesive system was applied following the manufacturer instructions. Groups I and III had a thin layer of primer applied on the tooth surface using disposable applicator Microbrush (Vigodente ${ }^{\circledR}$, Rio de Janeiro/ RJ-BR) and Group II used a simplified system that did not require primer application.

The bonding resin was inserted on the bracket base and the bracket was positioned on the tooth surface under pressure of 400 grams measured with the tensiometer (Morelli ${ }^{\oplus}$, Sorocaba/SP-BR) to standardize the adhesive layer thickness. The excess of the resin was removed using a small scaler (SS White ${ }^{\circledR}$, Rio de Janeiro/RJ-BR). In all of the groups, the specimens were light cured for 40 seconds using Optilight LD MAX (Gnatus ${ }^{\circledast}$, São Paulo/SP-BR).

A stainless steel device was made with a rectangular .021" X.025" wire (Morelli®, Sorocaba/SP-BR) to ensure that the bracket was perpendicular in a horizontal plane. Then, the teeth were fixed on the device by an elastomeric ring holding it to the wire. After fixation, the root was embedded in chemically cured acrylic resin and placed in metal rings. That way, the bonding surface was perpendicular to the horizontal plane and parallel to the direction of the force to be applied in the SBS test. All bonded specimens were stored in distilled water at $37^{\circ} \mathrm{C}^{16}$.

Twenty-four hours after the bonding procedures, the SBS test was performed using a Universal Testing Machine EMIC DL 2000 (Emic Equipamentos e Sistemas de Ensaio Ltda., São José dos Pinhais/PR-BR) at $0.5 \mathrm{~mm} / \mathrm{min}$ crosshead speed, until the bracket failure. As the bracket was moved, the required strength was calculated using TESC software version 3.01. The shear strength was measured as Newton $(\mathrm{N})$ divided by the bracket base area in $\mathrm{mm}^{2}$ and converted to Megapascal (Mpa).

Subsequently, the ARI was assessed with stereoscopic magnifying glass (722 Schwenningen, Waldmann-Leuchterz, Germany) under 20x magnification. A modification of the ARI originally proposed by Artun and Bergland ${ }^{17}$ was used. According to the modified ARI the following scores are assigned: score $\mathbf{0}$, no adhesive bonded 
on the bracket; score 1, 50\% of the adhesive bonded on the bracket; score 2, 75\% of the adhesive bonded on the bracket and score $\mathbf{3}$, the whole adhesive bonded on the bracket.

The values were entered into Excel for the statistical analysis. Analysis of Variance (ANOVA) was applied to estimate shear strength and verify statistically significant differences between groups I, II and III. The Tukey honestly significant difference (HSD) post hoc test was used to compare the group means. The Kruskal-Wallis test was applied to compare the ARI scores.

\section{Results}

The descriptive statistics for SBS in megapascals (MPa) is shown in Table 1. Statistically significant differences were observed among groups according to ANOVA. The Tukey test revealed that group III differed from groups I and II. No statistically significant differences between groups I and II were observed $(p<0.01)$.

Table 1. Descriptive statistics in Megapascals (MPa) of shear bond strengths of experimental groups studied

\begin{tabular}{lccc}
\hline Groups & $\mathbf{n}$ & Mean & Standard deviation \\
\hline Group I & 15 & $16.39 \mathrm{a}$ & 3.78 \\
\hline Group II & 15 & $18.08 \mathrm{a}$ & 8.50 \\
\hline Group III & 15 & $7.28 \mathrm{~b}$ & 2.51 \\
\hline
\end{tabular}

Different letters indicate significant difference by Tukey test $(P<0.001)$.

The ARI scores are presented in Table 2. The Kruskal-Wallis test showed no significant differences among groups regarding the ARI score $(p<0.05)$. Most specimens from groups presented ARI scores ranging from 2 to 3.

Table 2. Frequency distribution of adhesive remnant index (ARI) of groups studied

\begin{tabular}{llcccc}
\hline \multirow{2}{*}{ Groups } & \multicolumn{5}{c}{ ARI scores } \\
\cline { 3 - 5 } & & $\mathbf{0}$ & $\mathbf{1}$ & $\mathbf{2}$ & $\mathbf{3}$ \\
\hline GI & Frequency & $0(0 \%)$ & $0(0 \%)$ & $2(13.3 \%)$ & $13(86.7 \%)$ \\
\hline GII & Frequency & $0(0 \%)$ & $1(6.7 \%)$ & $5(33.3 \%)$ & $9(60 \%)$ \\
\hline GIII & Frequency & $0(0 \%)$ & $1(6.7 \%)$ & $1(6.7 \%)$ & $13(86.7 \%)$ \\
\hline
\end{tabular}

ARI - 0, no adhesive bonded on the bracket; $1: 50 \%$ of the adhesive bonded on the bracket; $2: 75 \%$ of the adhesive bonded on the bracket and 3: the whole adhesive bonded on the bracket. There were no significant differences among groups ( $P$-value $<0.05)$.

\section{Discussion}

The flat surface of human maxillary central incisor is ideal for studies on shear strength $^{18}$. However, improved health conditions for the population in general, advanced restoration techniques and greater access to health services have led to a 
decrease in the number of tooth extractions and a subsequent decrease in the offer of human teeth for studies in vitro. In our study, we used the bovine incisors because they are easily obtained, have low cost, and are similar to human teeth, which makes them an option to replace human teeth in research ${ }^{18}$.

Bonding orthodontic brackets onto tooth surface remains a debatable subject. A fast and effective bonding of orthodontic appliances is a clinical challenge regarding the use of adhesive systems since the procedures are generally carried out in humid environments with relative isolation of operative field. Therefore, it is important to apply simplified systems to reduce steps of the procedure and clinical time to ensure a secure bonding ${ }^{19}$.

The ideal properties of luting agents for orthodontic brackets are as follows: sufficient adhesive strength to tolerate orthodontic forces during treatment; fluidity required to penetrate the material during bonding; viscosity to maintain the bracket positioned before cement polymerization; proper time to reach the correct bracket positioning and remove the excess material; possibility of working in a humid environment to decrease posterior teeth deviation index, fluoride release and risks of white spots; removal with no damages to the enamel surface ${ }^{19}$.

The configuration of light cure adhesives requires primer and adhesive separately or unified. The configuration of Orthocem presents adhesive and primer unified. The mono-component configuration of bracket bonding reduces the process to only 2 steps. The Orthocem adhesive system presents the following characteristics: simplified technique, proper viscosity, the presence of fluoride, high shear strength, easy removal, and enamel polishing.

In this study, the Transbond XT adhesive system was used as a control group because it was shown to be efficient and exhaustively tested with proven characteristics to resist masticatory forces ${ }^{1,6,11-15}$.

Ryou et al. ${ }^{6}$ (2008) compared fluidic systems that do not require intermediate steps with Transbond XT observing that, although Transbond XT was superior to the other systems in terms of bond strength, their adhesive strength was sufficient, and therefore suitable for clinical application.

Although, there are studies that compared Orthocem or Orthobond to other adhesive systems ${ }^{1,10,11-15}$, in our knowledge these work would be the first one to compare these 2 adhesive systems.

The null hypothesis stating that there are no significant differences in SBS among the bonding materials was rejected. No statistically significant differences were observed between systems Transbond XT and Orthocem; however, both of them showed statistically significant increases in adhesive strength $(p<0.001)$ when compared to Orthobond.

In the present study, the mean adhesive strength of Orthocem (18.08 $\pm 8.5 \mathrm{MPa})$ was higher than the values reported in previous studies ${ }^{11,12}$ which were $8.31 \pm 3.5 \mathrm{MPa}$ and $4.2 \pm 0.8 \mathrm{MPa}$, respectively under the same conditions. The difference between these values is probably due to different bracket base mesh used. 
Our results for Orthobond were quite similar to those obtained by other researchers|13-15, who evaluated the SBS of the Orthobond system.

According to Reynolds ${ }^{3}$, the adhesive strength values between 5.9 and $7.8 \mathrm{MPa}$, which would be adequate to support masticatory forces, are acceptable for clinical use and show satisfactory performance. In our study, all of the experimental groups presented values compatible with clinical requirements.

In the present investigation, evaluation of the ARI scores showed no significant differences between groups I, II, and III. The ARI scores showed a predominance of scores 2 and 3 , which means that most of the adhesive remained attached to the bracket base and minimum amount remained on the enamel surface. Clinically, this implies that a shorter time is required to remove the adhesive after debonding. Our results for ARI scores are in agreement with some studies ${ }^{4,20}$ that state that the resin should remain on the bracket after the debonding, claiming it would require fewer steps to remove the adhesive from enamel ${ }^{4,20}$. Besides, it indicates that the cohesive strength of enamel is superior to the bond strength of bracket base. On the other hand, the residual adhesive remaining on enamel surface reduces enamel fractures; in addition, the residual adhesive is removed using special drill without any damage to tooth enamel ${ }^{21}$

In conclusion, both of the adhesive systems Orthocem and Transbond XT had higher SBS than Orthobond. All 3 systems examined presented similar enamel fracture pattern.

\section{References}

1. Busato MCA, Busato PMR, Dotto DV, Pedrotti S, Gasparello, CR. Evaluation of shear bond strength of brackets bonded with different orthodontic resins. Rev Clin Ortodon Dental Press. 2013 Apr-May;12(2):94-9. Portuguese

2. Newman GV. Epoxy adhesives for orthodontic attachments: progress report. Am J Orthod Dentofacial Orthop. 1965 Dec;51(12):901-12.

3. Reynolds IR. A review of direct orthodontic bonding. Br J Orthod. 1975;2:171-8.

4. Sorel O, El-Alam R, Chagnedy F, Gathelineau G. Comparison of bond strength between simple foil and laser structured base retention brackets. Am J Orthod Dentofac Orthop. 2002 Sep;122(3):260-6.

5. Chung $\mathrm{CH}$, Friedman SD, Mante FK. Shear bond strength of rebounded mechanically retentive ceramic brackets. Am J Orthod Dentofacial Orthop. 2002 Sep;122(3):282-7.

6. Ryou DB, Park HS, Kim KH, Kwon TY. Use of flowable composites for orthodontic bracket bonding. Angle Orthod. 2008 Nov; 78(6):1105-9. doi: 10.2319/013008-51.1.

7. Sant'Anna EF, Monnerat ME, Chevitarese $\mathrm{O}$, Stuani MB. Bonding brackets to porcelain in vitro study. Braz Dent J. 2002;13(3):191-6.

8. Tavares SW, Consani S, Nouer DF, Magnani MB, Nouer PR, Martins LM. Shear bond strength of new and recycled brackets to enamel. Braz Dent J. 2006;17(1):44-8.

9. Meehan PM, Foley TF, Mamandras AH. A comparison of the shear bond strengths of two glass ionomer cements. Am J Orthd Dentofacial Orthop. 1999 Feb;115(2):125-32.

10. Teixeira CM, Roya RR, Oliveira MT. Influence of variation on polymerization time in the shear bond strength for different cements to orthodontic brackets. Rev Bras Odontol. 2012 Jul/Dez;69(2):220-3. Portuguese. 
11. Scribante A, Sfondrini MF, Fraticelli D, Daina P, Tamagnone A, Gandini, P. The influence of no-primer adhesives and anchor pylons bracket bases on shear bond strength of orthodontic brackets. Biomed Res Int. 2013;2013:315023. doi: 10.1155/2013/315023.

12. Oliveira BLS, Costa, AR, Correr AB, Crepaldi MV, Correr-Sobrinho L, Santos JCB. Influence of adhesive and bonding material on the bond strength of bracket to bovine tooth. Braz J Oral Sci. 2017 Jul/Sep;16(3):1-7. e17033.

13. Pithon MM, Santos RL, Oliveira MV, Sant'anna EF, Ruellas ACO. Evaluation of the shear bond strength of the Orthobond composite under different conditions. RGO 2008 Oct/Dez;56(4):405-10. Portuguese.

14. Vilar RV, Souza, NF, Cal-Neto JP, Galvão M, Sampaio-Filho H, Mendes Ade M. Shear bond strength of brackets bonded with two light-curing orthodontic adhesives. J Adhes Dent. 2009 Aug;11(4):259-62.

15. Pithon MM, Santos RL, Oliveira MV, Sant'anna EF, Ruellas ACO. Evaluation of the shear bond strength of two composites bonded to conditioned surface with self-etching primer. Dental Press J Orthod 2011 Ar/Apr;16(2):94-9. doi: 10.1590/S2176-94512011000200012. Portuguese.

16. Cacciafesta V, Sfondrini MF, De Angelis M, Scribante A, Klersy C. Effect of water and saliva contamination on shear bond strength of brackets bonded with conventional, hydrophilic, and selfetching primers. Am J Orthod Dentofacial Orthop. 2003 Jun;123(6):633-40.

17. Artun J, Bergland S. Clinical trials with crystal growth conditioning as an alternative to acid-etch enamel pretreatment. Am J Orthod. 1984 Apr;85(4):333-40.

18. Oesterle LJ, Shellhart WC, Elanger GK. The use of bovine enamel in bonding studies. Am J Orthod Dentofacial Orthop. 1998 Nov;114(5):514-9.

19. Buonocore MG. A simple method of increasing the adhesion of acrylic filling materials to enamel surface. J Dent Res. 1955 Dec;34(6):849-53.

20. Costa AR, Correr AB, Puppin-Rontani RM, Vedovello SA, Valdrighi HC, Correr-Sobrinho $L$, et al. Effect of bonding material, etching time and silane on the bond strength of metallic orthodontic brackets to ceramic. Braz Dent J. 2012 Jul-Aug;23(3):223-7. doi: http://dx.doi.org/10.1590/S0103-64402012000300007.

21. MacColl GA, Rossouw PE, Titley KC, Yamin C. The relationship between bond strength and orthodontic bracket base surface area with conventional and microetched foil-mesh bases. Am J Orthod Dentofacial Orthop. 1998 Mar;113(3):276-81. 